\title{
Nine new open clusters within 500 pc from the Sun
}

\author{
Siegfried Röser ${ }^{1}$, Elena Schilbach ${ }^{1}$, and Bertrand Goldman ${ }^{2,3}$ \\ 1 Zentrum für Astronomie der Universität Heidelberg, Mönchhofstraße 12-14, 69120 Heidelberg, Germany \\ e-mail: [roeser; elena] @ari .uni-heidelberg.de \\ 2 Max-Planck-Institut für Astronomie, Königstuhl 17, 69117 Heidelberg, Germany \\ 3 Observatoire astronomique de Strasbourg, Université de Strasbourg - CNRS UMR 7550, 11 rue de l'Université, 67000 Strasbourg, \\ France
}

Received 21 June 2016 / Accepted 6 August 2016

\begin{abstract}
Aims. One of the results of the Milky Way Star Clusters (MWSC) survey was the detection of a slight under-density of old (ca. 1 Gyr) clusters within the nearest kilo-parsec from the Sun. This under-density may be due to an ineffectiveness in the detection of larger structures with lower surface brightness. We report on our attempts to reveal such clusters.

Methods. We derived proper motions from a combination of Tycho-2 with URAT1, and obtained a mean precision of about 1.4 mas/yr per co-ordinate for 1.3 million stars north of $-20^{\circ}$ declination. We cut the sky into narrow proper motion slices and searched for spatial over-densities of stars in each slice. We then examined stars from over-densities in optical and near-infrared colour-magnitude diagrams to determine if they are compatible with isochrones of a cluster. We estimated the field star contamination using our data and the Besançon Galactic model.

Results. We detected nine hitherto unknown open clusters in the vicinity of the Sun with ages between 70 Myr and 1 Gyr, and distances between 200 and $500 \mathrm{pc}$.
\end{abstract}

Key words. open clusters and associations: general - catalogs - surveys - proper motions

\section{Introduction}

Star clusters are considered building blocks of the Galaxy. Their study is important for our understanding of star formation and of the history of the Milky Way. The Milky Way Star Clusters (MWSC) project (Kharchenko et al. 2012) aims to deliver a sample of open clusters within the larger solar neighbourhood, which covers a representative data set of clusters of all ages. Although Kharchenko et al. (2013) found that their sample of some 2800 open clusters is essentially complete to $1.8 \mathrm{kpc}$ from the Sun, for a small subset of the oldest open clusters $(\log t \geq 9)$, these authors noted evidence of incompleteness within about $1 \mathrm{kpc}$ from the Sun (see Fig 4. in Kharchenko et al. 2013).

In the following years, several efforts were undertaken to fill the gap of old clusters within $1 \mathrm{kpc}$. A classical method to reveal new open clusters is searching for stellar over-densities on the sky. This method has been applied by Schmeja et al. (2014) on the basis of the 2MASS catalogue (Skrutskie et al. 2006). Although they found 139 new open clusters, the situation within $1 \mathrm{kpc}$ did not change. The depth of 2MASS is responsible for a high average background noise, so this method turned out to be more effective for the detection of compact clusters which, in general, are the more distant clusters.

For a better detection of over-densities, the background noise can be reduced by using proper motions to construct subsets of co-moving stars. Open clusters are gravitationally bound entities of co-eval stars, so common motion is a necessary condition for stars forming an open cluster. The internal velocity dispersion depends on cluster mass and usually reaches a few hundred meters per second for the vast majority of open clusters in the Galaxy. For example, the internal one-dimensional velocity dispersion found to be $0.44 \mathrm{~km} \mathrm{~s}^{-1}$ for an old open cluster such as the Hyades with a tidal mass of $275 M_{\odot}$ (see Röser et al. 2011). Even for loose ensembles, such as nearby young moving groups, the one-dimensional velocity dispersions are about $1 \mathrm{~km} \mathrm{~s}^{-1}$ (see Mamajek 2016). At 200 pc, 1 mas/y in proper motion roughly corresponds to $1 \mathrm{~km} \mathrm{~s}^{-1}$ in space motion, so the internal velocity dispersion of an open cluster, and even of a moving group should still be smaller than the typical present-day accuracy of proper motions of stars at distances larger than about 200 pc.

In their search for undetected clusters, Scholz et al. (2015) used proper motions from a subset of 399 million stars of the PPMXL (Röser et al. 2010) catalogue. The accuracy of the proper motions in PPMXL depends essentially on declination. For stars with 2MASS observations, Röser et al. (2010) give a typical accuracy of proper motions of 4 mas/yr north of $-30^{\circ}$ declination, and 9 mas/yr south of it. Taking this into account, Scholz et al. (2015) divided the catalogue into 441 circular proper motion bins (radius 15 mas/yr) within \pm 50 mas/yr in $\mu_{\alpha} \cos \delta$ and in $\mu_{\delta}$. In each proper motion bin, they looked for over-densities in small sky areas ("sky pixels") of $0.25 \times$ $0.25 \mathrm{deg}^{2}$, which corresponds to the typical size of clusters in the MWSC survey. As a result, 63 new open clusters were found by this approach, however, all were farther away from the Sun than $1 \mathrm{kpc}$.

Why did Scholz et al. (2015) not find new clusters within $1 \mathrm{kpc}$ ? Possibly, the missing clusters (within $1 \mathrm{kpc}$ ) should have a larger angular extent on the sky than is reflected in the small sky pixels chosen by Scholz et al. (2015). Also, the large bins (a radius of $15 \mathrm{mas} / \mathrm{yr}$ ) in proper motion space do not efficiently reduce the sky background, which makes over-densities more difficult to be detected. For the detection of nearby clusters small (less than about 1 mas/yr) proper motion bins and large spacial bins of about $1 \mathrm{deg}^{2}$ could be a reasonable choice. 
The forthcoming Gaia data releases will be ideal for this purpose. However, in this paper we try to show that even a moderate progress in the precision of proper motions allows us to reveal so far unknown open clusters within $1 \mathrm{kpc}$ from the Sun. The results presented here should be looked at as a current snapshot of what we can expect from Gaia data. We note that it was not our intention to determine the most accurate astrophysical parameters of these clusters on the basis of the presently available astrometric and photometric data as these parameters will be revised within a short time using the Gaia results.

In Sect. 2 we describe how we improved the Tycho-2 (Høg et al. 2000) proper motions and then explain the adopted method of cluster detection in Sect. 3. In Sect. 4 we present the newly found clusters, and the discussion in Sect. 5 concludes the paper.

\section{Improving Tycho-2 proper motions}

We used URAT1 (Zacharias et al. 2015) to improve the Tycho-2 proper motions and to test what proper motions, which are more precise than those of Tycho-2, can do for open cluster studies. URAT1 contains 228 million objects down to about $R=$ $18.5 \mathrm{mag}$, north of about $-20^{\circ}$ declination. For the bulk of the Tycho-2 stars, URAT1 gives positions at a mean epoch around 2013.5 and an accuracy level of about 20 mas per co-ordinate. We cross-matched URAT1 with Tycho-2 (the original data set tyc2.dat from CDS), and obtained new proper motions via a least-squares adjustment as described, for example in PPMXL (Röser et al. 2010). To avoid formally ultra-precise astrometry for a small number of stars, we chose a 10 mas floor for the precision of a URAT1 position.

We constructed a catalogue, hereafter called TYCURAT, containing 1300000 stars north of $-20^{\circ}$ declination with an average formal precision of $1.35 \mathrm{mas} / \mathrm{yr}$ in proper motion, both in right ascension and in declination. The corresponding accuracy in Tycho-2 is 2.37 mas/yr, which gives a formal improvement of proper motion accuracy of $40 \%$. URAT1 was astrometrically calibrated by its authors using UCAC4 (Zacharias et al. 2013), which is nominally on the ICRS system. No attempt has been made to investigate potential regional systematic distortions in the proper motion system of the new catalogue TYCURAT. These possible zonal deviations are not crucial for a potential detection of open clusters and moving groups on areas of a few square degrees. We then cross-matched the TYCURAT catalogue with ASCC-2.5 (Kharchenko 2001) to obtain the Johnson $B, V$ and 2MASS $J, H, K_{\mathrm{S}}$ photometry, which is included in the latter ${ }^{1}$.

\section{Detecting nearby stellar groups}

The stellar content of TYCURAT is a subset of the ASCC-2.5 catalogue, which was already used for a systematic search of open clusters by Kharchenko et al. (2005). These authors revealed 109 new Galactic open clusters by looking around each star (seed) brighter than $V=9.5$ in an area less than 0.3 deg to see if they could find companions with proper motions within $1 \sigma$ of the proper motion of the seed. In a next step, they checked whether the selected stars fit to an isochrone in an $(B-V, V)$ colour-magnitude diagram (CMD). Using TYCURAT, which has the same stellar content on 67 per cent of the sky, we could not expect to find a large number of new clusters. However, with

\footnotetext{
1 TYCURAT is available on request from roeser@ari.uni-heidelberg.de
}

improved proper motions and changing the detection criteria we get a chance to reveal those clusters that escaped the detection by Kharchenko et al. (2005).

\subsection{Astrometric steps}

In our approach, we first start with a narrow range of 1.5 mas/yr in proper motion space to down-select stars with the same proper motion and look for their concentrations in circular bins with a radius of $0.5 \mathrm{deg}$ on the sky to reveal cores of possible co-moving objects. The details of the procedure are described below.

(a) Depending on their proper motions, we divided TYCURAT stars into subsets (which we call proper motions slices) by the following rule. In the proper motion plane $\left(\mu_{\alpha} \cos \delta, \mu_{\delta}\right)$ we selected a quadratic area around the origin given by the condition $\left|\mu_{\alpha} \cos \delta\right|+\left|\mu_{\delta}\right| \leq 43$ mas/y and chose grid points (centres) at each integer value $(i, k)$. This gives 3785 proper motion slices. A slice $(i, k)$ then contains all stars for which $\left(\mu_{\alpha} \cos \delta-i\right)^{2}+\left(\mu_{\delta}-k\right)^{2} \leq 1.5^{2}$ holds. The number of stars per slice strongly varies and depends on the total proper motion. The maximum number of 44000 stars is at $\left(\mu_{\alpha} \cos \delta, \mu_{\delta}\right)=$ $(-1,-3)$, whereas the number of stars is less than 150 at the borders of our square. About $90 \%$ of all slices contain less than 6000 stars. These slices represent a seven-fold overlap of the proper motion plane, with the effect that the same overdensity on the sky may appear in neighbouring slices, but not necessarily with the same signal-to-noise ratio.

(b) In the second step we searched for over-densities in the proper motion slices. For each star in a proper motion slice, we counted all its neighbours within a radius of $0.5 \mathrm{deg}$ (called sky circle) and determined the surface density $S$ (stars/square degree). Surrounding each star's sky circle we chose an area of 9 sq. deg, where we determined the background surface density $B$. We only retain stars if $S / B$ of their sky circle is larger than 3.0 and their $S-B$ is larger than 5 per square degree. If the angle between two stars is less than $0.5 \mathrm{deg}$, each of them is in the other's sky circle. We discard the one with the lower $S / B$. This cleaning goes on until only "isolated" sky circles are left, which we call Level-0 centres.

As our system of proper motion slices represents a seven-fold overlap of the sky, a Level- 0 centre in slice $n$ could have the same or a neighbouring Level- 0 centre in slice $m$. If they are within the radius of a sky circle and the difference of their actual proper motions is less than $1.5 \mathrm{mas} / \mathrm{yr}$, only the Level- 0 centre with higher $S / B$ is retained. These survivors are called Level-1 centres; they are disjunct and are possible candidates of new open clusters.

Of course, many of the Level-1 centres could be related to known open clusters. Therefore, we cross-matched them with the MWSC clusters (Kharchenko et al. 2013) and with the most recent cluster list DAML02 VERSION 3.5 of 2016 Jan. 28 (Dias et al. 2002). We discarded all the Level-1 centres within $0.7 \mathrm{deg}$ of a known cluster, if the radius of the known cluster was larger than $0.2 \mathrm{deg}$. If the radius of a cluster in MWSC or in DAML is smaller than $0.2 \mathrm{deg}$, it cannot be identical to one of the objects found here. About 200 Level-1 centres surviving this process (called Level-2 centres) were then subject to a photometric check of their existence as clusters.

\subsection{Photometric steps.}

(a) We first made a quick and coarse check in the $\left(J-K_{\mathrm{S}}, K_{\mathrm{S}}\right)$ CMD of the stars in a sky circle around a Level-2 centre 
Table 1. New open clusters.

\begin{tabular}{|c|c|c|c|c|c|c|c|c|c|c|c|c|}
\hline Name & $N$ & $\begin{array}{l}l \\
\operatorname{deg}\end{array}$ & $\begin{array}{c}b \\
\operatorname{deg}\end{array}$ & $\begin{array}{l}\text { RAc } \\
\text { deg }\end{array}$ & $\begin{array}{l}\text { Dec } \\
\text { deg }\end{array}$ & $\begin{array}{r}\overline{\mu_{\alpha} \cos \delta}(\mathrm{rms}) \\
\text { mas/yr }\end{array}$ & $\begin{array}{l}\overline{\mu_{\delta}}(\mathrm{rms}) \\
\text { mas/yr }\end{array}$ & $\begin{array}{l}D \\
\mathrm{pc}\end{array}$ & $\log t$ & $\begin{array}{l}Z \\
\mathrm{pc} \\
\end{array}$ & $\begin{array}{c}E(B-V) \\
\quad \text { mag }\end{array}$ & $\begin{array}{c}\text { Dmod } \\
\text { mag }\end{array}$ \\
\hline RSG1 & 15 & 167.8 & -2.6 & 75.57 & 37.56 & $0.20(0.13)$ & $-12.32(0.13)$ & 324 & 8.10 & -15 & 0.24 & 7.55 \\
\hline RSG2 & 23 & 162.4 & 26.2 & 110.57 & 54.81 & $-2.41(0.13)$ & $-29.54(0.18)$ & 200 & 8.10 & 88 & 0.04 & 6.50 \\
\hline RSG3 & 19 & 232.0 & 16.1 & 126.12 & -8.81 & $-7.98(0.15)$ & $4.33(0.15)$ & 437 & 9.00 & 121 & 0.01 & 8.20 \\
\hline RSG4 & 16 & 87.8 & 19.5 & 288.54 & 56.96 & $-0.27(0.16)$ & $5.71(0.17)$ & 363 & 8.55 & 121 & 0.07 & 7.80 \\
\hline RSG5 & 45 & 81.8 & 6.0 & 303.67 & 45.59 & $3.41(0.09)$ & $2.22(0.09)$ & 355 & 7.70 & 37 & 0.04 & 7.75 \\
\hline RSG6 & 54 & 78.9 & 0.6 & 307.49 & 40.08 & $5.86(0.11)$ & $1.97(0.10)$ & 347 & 7.80 & 3 & 0.04 & 7.70 \\
\hline RSG7 & 21 & 108.4 & -0.8 & 343.94 & 58.73 & $5.74(0.13)$ & $-0.42(0.16)$ & 457 & 8.30 & -7 & 0.06 & 8.30 \\
\hline RSG8 & 19 & 108.8 & -0.7 & 344.56 & 59.06 & $5.81(0.19)$ & $-0.70(0.13)$ & 331 & 8.50 & -4 & 0.05 & 7.60 \\
\hline RSG9y & 13 & 155.1 & 5.0 & 72.74 & 52.23 & $8.39(0.18)$ & $-15.23(0.19)$ & 224 & 7.50 & 19 & 0.05 & 6.75 \\
\hline RSG9o & 13 & 155.1 & 5.0 & 72.74 & 52.23 & $8.39(0.18)$ & $-15.23(0.19)$ & 224 & 9.00 & 19 & 0.02 & 6.75 \\
\hline
\end{tabular}

using TOPCAT $^{2}$ (Taylor 2005). A Level-2 centre was selected for a more careful treatment if stars with $(J-K \mathrm{~s})<$ 0.4 showed a tendency towards building a part of a main sequence or a turn-off region. The Level-2 centres were immediately rejected if, on the CMDs, their stars showed a random distribution, mostly at the limiting magnitude of Tycho-2. So, we could quickly check the Level-2 centres found in the previous step.

(b) Only 11 Level- 2 centres made it into the final process. From the stars in each Level- 2 centre's sky circles, we determined a mean position $\bar{\alpha}, \bar{\delta}$ and mean proper motions $\left(\overline{\mu_{\alpha} \cos \delta}, \overline{\mu_{\delta}}\right)$. Then we selected an area of 2 deg radius around $\bar{\alpha}, \bar{\delta}$ containing the stars from a proper motion slice around the mean proper motion.

The photometric $B, V$ data from ASCC-2.5 are pretty accurate for stars brighter than $V=10$, but the accuracy decreases rapidly for fainter stars. Therefore, we cross-matched the stars around each Level-2 centre with APASS9 (Henden et al. 2016) to obtain more precise optical photometry for fainter stars, knowing that there may be small systematic differences between the photometric systems of ASCC-2.5 and APASS9.

In order to determine membership, distance modulus, age, and reddening, we used Padova CMD $2.8^{3}$ solar metallicity isochrones both within the optical $(B-V, V)$ and the near-infrared $\left(J-K_{\mathrm{S}}, K_{\mathrm{S}}\right)$ CMDs. Allowing for possible binarity, we consider a star as a probable member if its offset from the isochrone is less than 2.5 sigma of its photometric error in magnitude and colour in both CMDs.

\section{Newly found open clusters}

For 8 out of 11 Level-2 centres, representing nine clusters (see below), we obtained reasonable fits to Padova isochrones. The newly detected clusterings are located in the solar neighbourhood at distances below $500 \mathrm{pc}$ from the Sun. The candidates RSG1 to RSG8 are very probably genuine physical groups. Membership and astrophysical parameters could be determined sufficiently well. Nevertheless, accurate parallaxes of at least several reliable cluster stars could improve the quality of parameter determination. A definite age cannot be derived for RSG9; this critically depends on the secure membership status of the two brightest stars.

Table 1 summarises the astrophysical parameters of the newly found objects. Columns 1 and 2 contain the names of the

\footnotetext{
2 Tool for OPerations on Catalogues And Tables

3 http://stev.oapd.inaf.it/cgi-bin/cmd
}

clusters and the number of probable members $(\mathrm{N})$, contained in TYCURAT, as determined in Sect. 3.2. All the following cluster parameters are determined from these probable members.

The coordinates of the centres in Galactic longitude $(l)$, Galactic latitude $(b)$, right ascension (RAc), and declination (Dec) are given in Cols. 3 to 6. In Cols. 7 and 8 lists the mean proper motion and the precision of the mean motions. The distances $D$, ages $(\log t)$, the distance $Z$ from the Galactic plane, reddening $E(B-V)$ and distance modulus (Dmod) are given in Cols. 9-13. The CMDs of the new clusters are shown in Fig. 1. To evaluate a possible contamination by the stellar population from the general Galactic field we used the Besançon galactic model (Robin et al. 2003) with realistic extinction included in the simulation, based upon the $3 \mathrm{D}$ extinction maps of Schlafly et al. (2014). As our underlying survey, the Tycho2 catalogue is about $90 \%$ complete down to $V=11.5$ (Høg et al. 2000) and rapidly gets incomplete beyond this value; we cut our simulations at this magnitude. For each cluster we simulated the contribution of field stars in the same proper motion range and on an area of $\approx 12.5 \mathrm{sq}$. deg around the centre and show the results in the CMDs of Fig. 2. The number of the predicted stars was always fairly less than the number of the actual stars in the cluster areas, a hint at the reality of the over-densities found.

Each of the nine clusters is briefly discussed below:

\section{RSG1}

The combined use of optical and NIR photometric data permitted a reliable selection of the most probable members. A parallax of $3.8 \pm 0.7$ mas (van Leeuwen 2007) and a spectral type B9 (Skiff 2014) of the brightest member (HD 32270) are consistent with the distance and age determined for RSG1. The members form a relatively compact group of $1 \times 1 \mathrm{sq}$. deg $(5.6 \mathrm{pc} \times 5.6 \mathrm{pc})$ of young stars moving with a significant tangential velocity of $19 \mathrm{~km} \mathrm{~s}^{-1}$ relative to the Sun. As seen from Fig. 2 the predicted field-star contamination is negligible for this cluster.

\section{RSG2}

RSG2 is the nearest cluster with the largest proper motion. This cluster groups around the two B8/B9 stars 19 Lyn A and 19 Lyn B, and includes three probable members with a relative parallax accuracy from $11 \%$ to $15 \%$ in van Leeuwen (2007). They indicate a mean distance of $197 \mathrm{pc}$, coinciding well with our determination based on isochrone fitting. Since RSG2 is so close to us, we allowed a search for possible members on a larger area of the sky $(R<3 \mathrm{deg})$. RSG2 is about as old as the Pleiades but is considerably less populated. Remarkably, it is located at about the same galactic longitude $\left(l=166^{\circ}\right.$ for RSG2 and $l=163^{\circ}$ for the Pleiades), but north of the Galactic plane 

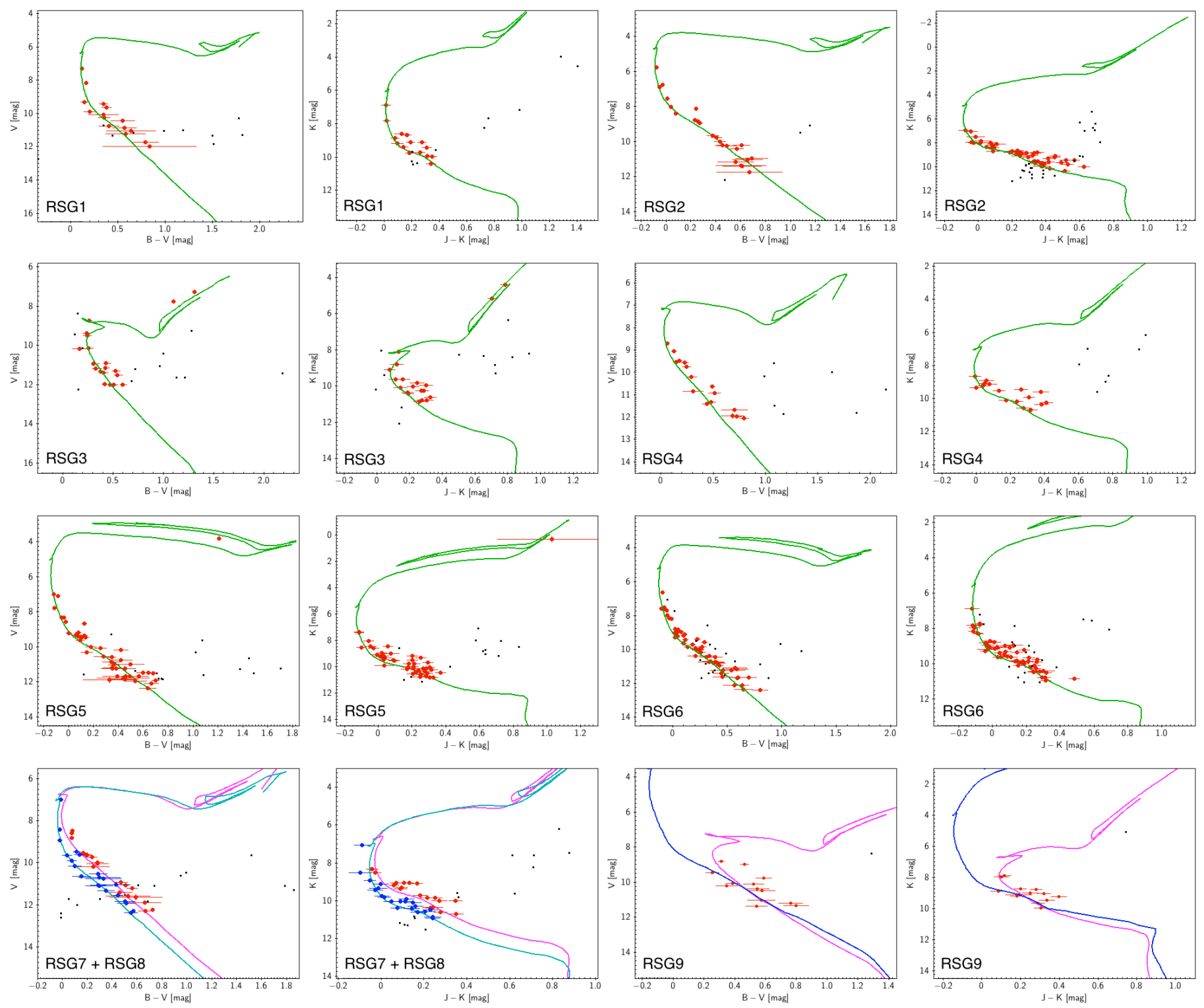

Fig. 1. Colour-magnitude diagrams $(B-V, V)$ and $\left(J-K_{\mathrm{S}}, K_{\mathrm{S}}\right)$ of the clusters RSG1 to RSG9 from Table 1. Thick red (blue in the case of RSG7) dots with error bars are confirmed photometric members; small black dots are field stars within the same proper motion slice within a 2 deg radius (a 3 deg radius in the case of RSG2) around the cluster centre. Fitted Padova isochrones corresponding to the ages given in Table 1 are shown as coloured lines.

( $b=26^{\circ}$ for RSG2 and $b=-23^{\circ}$ for the Pleiades). Because of its large proper motion, this cluster is practically free from field-star contamination (two stars obtained from the model). We could only show where posible contaminants would lie in the CMD in Fig. 2 when we used a large area of 200 sq. deg (factor seven in area).

\section{RSG3}

This cluster is rather far away to have accurate trigonometric parallaxes. According to van Leeuwen (2007), the two brightest members, HD 70358 (K1 III) and HD 70407 (K2 III), are located at distances between 270 and 480 pc from the Sun, which does not contradict our estimate from isochrone fitting. RSG3 is the oldest cluster in our sample and it represents a rather loose group of co-moving stars distributed within an area of $20 \mathrm{pc} \times 20 \mathrm{pc}$. In the case of RSG3, the stellar population from the Besançon model illuminates the problem of revealing old clusters in a shallow sky survey. The model gives a contamination of about $30 \%$ near the isochrone of the cluster. More accurate astrometric data from the first Gaia data release will decide upon the fate of this cluster.

\section{RSG4}

With an age of about $350 \mathrm{Myr}$, this cluster is a typical representative of the cluster population in the solar neighbourhood (see Piskunov et al. 2006). A parallax of $3.5 \pm 0.7$ mas of the brightest member HD 180007 (A0/A0III) is consistent with our estimate of the cluster distance. According to the model, the contamination is rather small, so we have no doubt about its existence.

RSG5

This cluster is the youngest one in our sample. There are four probable members with parallaxes measured by HIPPARCos with a relative accuracy of $10-20 \%$. The resulting average distance of about 325 pc $(275-435$ pc) is consistent with our estimate. The brightest star (31 Cyg) is a spectroscopic binary K4Ib+B4V (Pourbaix et al. 2004) with an age of $39.8 \pm 10.3$ Myr (Tetzlaff et al. 2011), which also supports our age estimate for the cluster. In accordance with the 

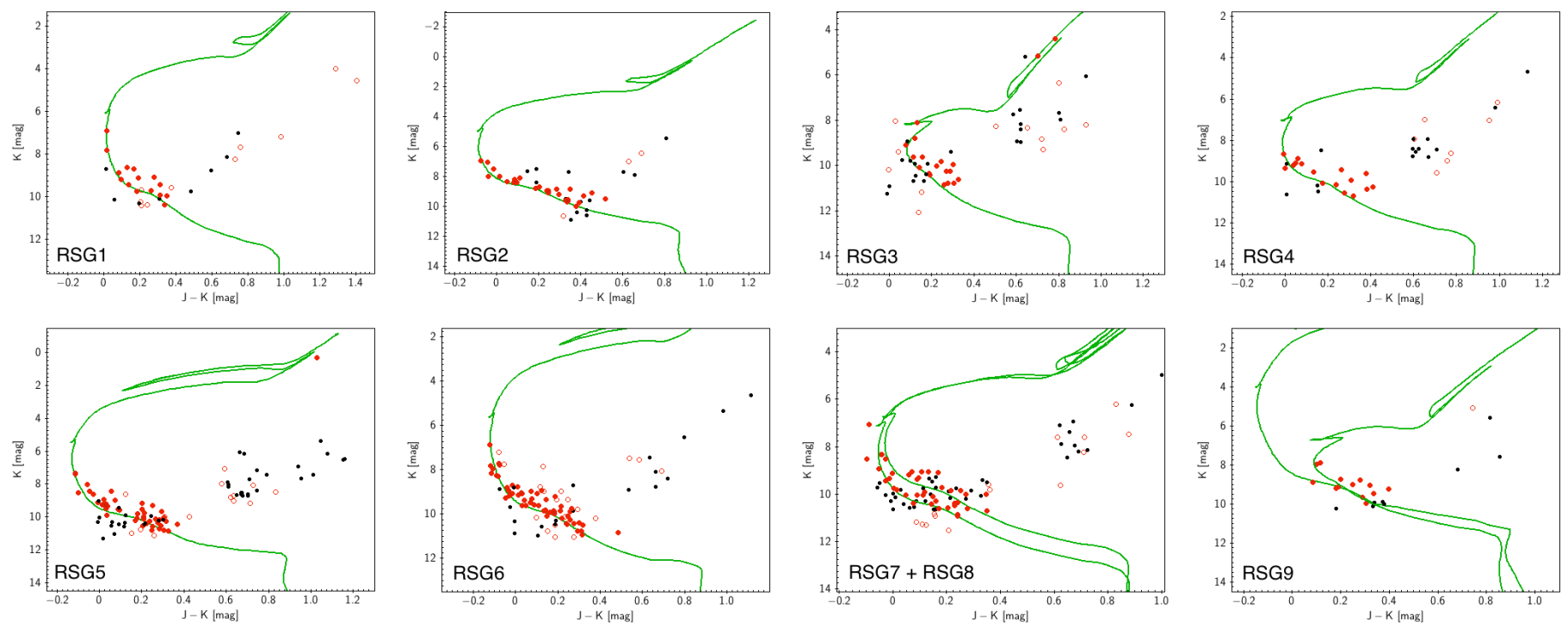

Fig. 2. Observed and predicted (by the Besançon model) $\left(J-K_{\mathrm{S}}, K_{\mathrm{S}}\right)$ CMDs in the areas of the clusters from Table 1 . Red symbols refer to observations, where thick dots indicate probable members and circles indicate field stars. The black dots are predicted field stars from the Besançon model of the Galaxy.

prediction from the model, the contamination by field stars is only about $15 \%$.

\section{RSG6}

As a relatively young, nearby, and well-populated cluster, RSG6 is concentrated within an area of about $2.5 \times 2.5$ sq. deg, $(15 \mathrm{pc} \times 15 \mathrm{pc})$ partly covering the cluster Roslund 6 (Roslund 1960). Roslund 6 is more distant, $544 \mathrm{pc}$; older, $\log t=8.67$; and smaller, radius $0.3 \mathrm{deg}$ (data from Kharchenko et al. 2013). There are HIPPARCOS parallaxes of a relative accuracy better than $18 \%$ for five B stars that were selected as probable RSG6 members. They place the cluster at a distance of about $330 \mathrm{pc}$ (230-450 pc), which coincides well with our distance estimate. The spectral type of the brightest star HD 194789, B6 IV/V (Skiff 2014) indicates a young age for RSG6. As RSG6 and Roslund 6 have nearly the same proper motions (given the precision in TYCURAT) the question of whether Roslund 6 is either a separate cluster behind RSG6 or a part of a large, complex RSG6 can only be solved definitely with Gaia parallaxes and proper motions. In Fig. 3 we show the area surrounding Roslund 6 and RSG6 on the sky. According to the model the contamination is less than $10 \%$, so we have no doubt about its existence.

RSG7 and RSG8

The CMDs of the co-moving stars in this sky area reveal a welldefined sequence, although it is too wide to be caused by binarity of several members. A possible explanation is that there is a mixture of several clusterings with about the same proper motions, but at different distances (one behind the other) projected on the sky in the same direction. We fit the observed distribution by two different isochrones; the results are given in Table 1 and Fig. 1. Unfortunately, the parallax was measured by HIPPARCos only for the brightest star with a relative accuracy better than $20 \%$ resulting in a distance between 355 and 500 pc. The Besançon model for the area of RSG7 and RSG8 in Fig. 2 shows moderate contamination near the sequences of the two clusters. With Gaia data a clear decision can be made about the number of groups and whether the stars are members of RSG7 or RSG8.

RSG9

This group is represented by only 13 co-moving stars distributed over an area of $5 \mathrm{pc} \times 10 \mathrm{pc}$ (with 8 stars concentrated to an

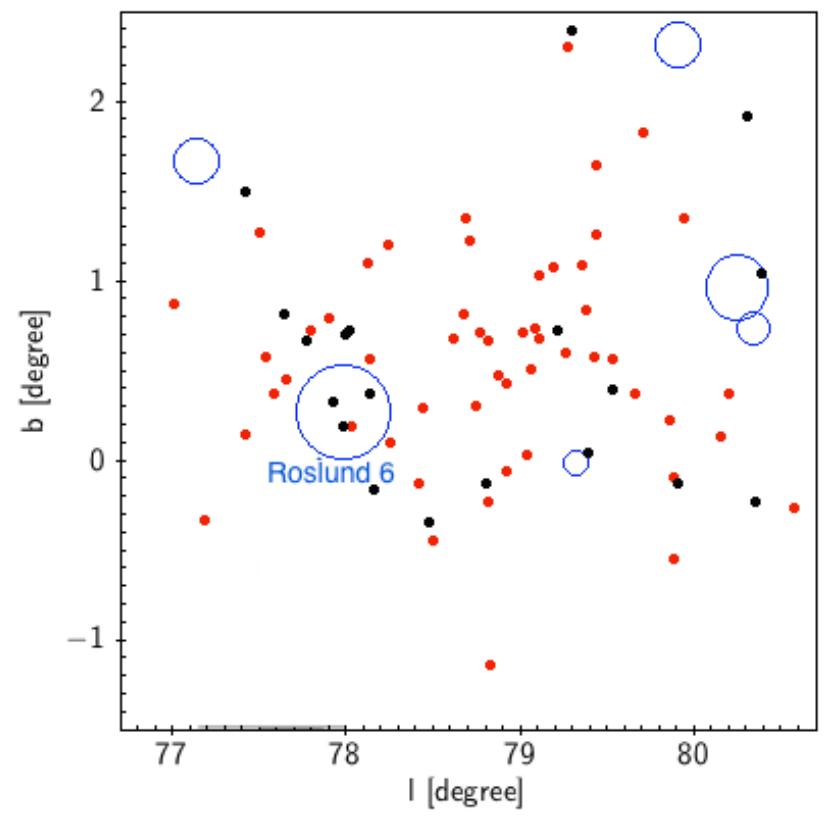

Fig. 3. Area around the new cluster RSG6 (galactic coordinates). Red dots indicate the probable members in RSG6; black dots indicate the TYCURAT stars within the same proper motion slice. Open clusters from MWSC are shown as large blue circles, the radius corresponding to the cluster radius $\mathrm{r} 2$ in MWSC.

inner core of $2 \mathrm{pc} \times 4 \mathrm{pc}$ ) and with a relatively large proper motion. The major axis of the cluster core is parallel to the galactic plane and the direction of the proper motion vector coincides with the major axis. For one star a parallax of $3.63 \pm 0.94$ mas (van Leeuwen 2007) was measured by HIPPARcos. Although the relative accuracy is rather moderate, this agrees with our distance estimate for RSG9. The age, however, cannot be determined definitely, i.e., all ages between $\log t=7.5$ and 9.0 are still acceptable. Here, Gaia is needed for a conclusive membership determination. On the other hand, the contamination by field stars is not an issue for this cluster. 


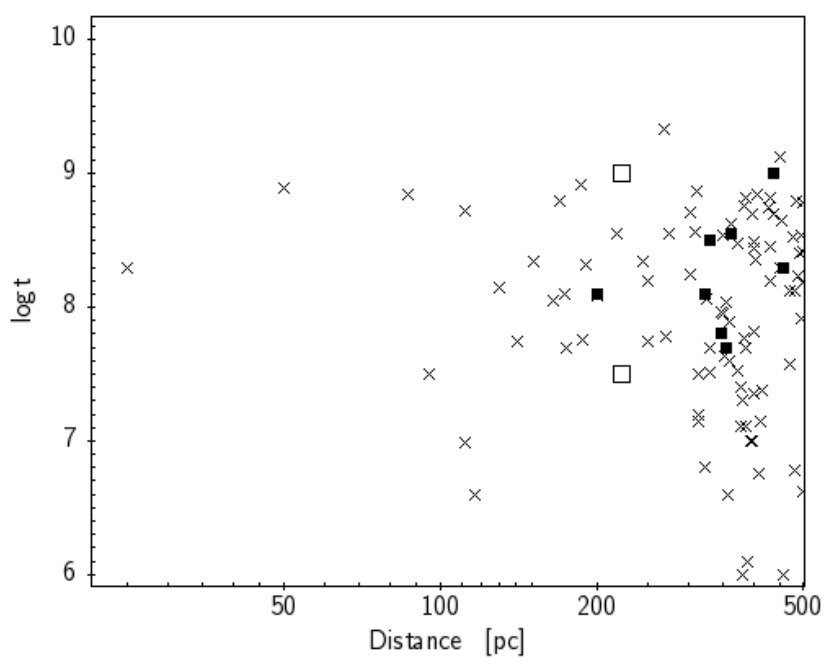

Fig. 4. Distances and ages of the newly found clusters within 500 pc. The new clusters are shown as black squares, whereas the MWSC clusters (Kharchenko et al. 2013) are shown as crosses. The open squares indicate the age spread of the cluster RSG9.

\section{Discussion}

Our results show that with highly precise proper motions and carefully selected search parameters it is still possible to reveal hitherto unknown open clusters even in the closer neighbourhood of the Sun up to 500 pc. On the other hand, we detected no new open cluster beyond that distance. A possible reason is the low limiting magnitude of Tycho-2. So, the larger the distance modulus and extinction, the less probable it becomes to obtain enough unevolved stars to find a cluster main sequence in the CMDs. It looks as if this happens at distance modulus 8.5 (roughly $500 \mathrm{pc}$ ).

Owing to the shallow depth of Tycho-2, we were only able to find the brightest and most massive stars in our clusters. Typical stellar mass functions in open clusters (see e.g. Fig. 3 in Bastian et al. 2010) imply that the total mass of a cluster is governed by stars around $0.5 M_{\odot}$ which, at distances prevailing here, are below the limiting magnitude of Tycho-2. Not knowing the mass we cannot make a statement about whether the new clusters are still gravitationally bound or if they are dissolving.

Our findings contribute substantially to the open cluster population within 500 pc from the Sun. In Fig. 4 we show the distribution of the distances and ages of our nine newly found clusters. The clusters are shown as black squares, and only cluster RSG9 is presented as two open squares indicating the range of possible ages. Also shown are the 92 clusters (crosses) from Kharchenko et al. (2013). Although we could only search $67 \%$ of the sky, our results increase the total number of clusters within
500 pc by $10 \%$. Only two of the MWSC clusters, Ruprecht 147 and NGC 752, have $\log t \geq 9$. RSG3 adds one more cluster to this old cluster generation; RSG9 may add another one if its old age is confirmed.

The first Gaia data release will contain TGAS (Michalik et al. 2015) supplying proper motions similar to or better than TYCURAT on the whole sky. But the biggest progress will be achieved by the about 2 million absolute parallaxes. These will surpass the HIPPARcos parallaxes in quality and by a factor of 20 in quantity. It will open the path to a substantial leap in open cluster research.

Acknowledgements. This study was supported by Sonderforschungsbereich SFB 881 "The Milky Way System" (subprojects B5 and B7) of the German Research Foundation (DFG). It is a great pleasure to acknowledge Mark Taylor from the Astrophysics Group of the School of Physics at the University of Bristol for his wonderful work on TOPCAT, Tool for OPerations on Catalogues And Tables. We made extensive use of the major functions of this beautiful tool. This research has made use of the SIMBAD database and of the VizieR catalogue access tool, operated at CDS, Strasbourg, France.

\section{References}

Bastian, N., Covey, K. R., \& Meyer, M. R. 2010, ARA\&A, 48, 339

Dias, W. S., Alessi, B. S., Moitinho, A., \& Lépine, J. R. D. 2002, A\&A, 389, 871 Henden, A. A., Templeton, M., Terrell, D., et al. 2016, VizieR Online Data Catalog: II/336

Høg, E., Fabricius, C., Makarov, V. V., et al. 2000, A\&A, 355, L27

Kharchenko, N. V. 2001, Kinematika i Fizika Nebesnykh Tel, 17, 409

Kharchenko, N. V., Piskunov, A. E., Röser, S., Schilbach, E., \& Scholz, R.-D 2005, A\&A, 440, 403

Kharchenko, N. V., Piskunov, A. E., Schilbach, E., Röser, S., \& Scholz, R.-D. 2012, A\&A, 543, A156

Kharchenko, N. V., Piskunov, A. E., Schilbach, E., Röser, S., \& Scholz, R.-D. 2013, A\&A, 558, A53

Mamajek, E. E. 2016, in IAU Symp. 314, eds. J. H. Kastner, B. Stelzer, \& S. A. Metchev, 21

Michalik, D., Lindegren, L., \& Hobbs, D. 2015, A\&A, 574, A115

Piskunov, A. E., Kharchenko, N. V., Röser, S., Schilbach, E., \& Scholz, R.-D. 2006, A\&A, 445, 545

Pourbaix, D., Tokovinin, A. A., Batten, A. H., et al. 2004, A\&A, 424, 727

Robin, A. C., Reylé, C., Derrière, S., \& Picaud, S. 2003, A\&A, 409, 523

Röser, S., Demleitner, M., \& Schilbach, E. 2010, AJ, 139, 2440

Röser, S., Schilbach, E., Piskunov, A. E., Kharchenko, N. V., \& Scholz, R.-D. 2011, A\&A, 531, A92

Roslund, C. 1960, PASP, 72, 205

Schlafly, E. F., Green, G., Finkbeiner, D. P., et al. 2014, ApJ, 789, 15

Schmeja, S., Kharchenko, N. V., Piskunov, A. E., et al. 2014, A\&A, 568, A51

Scholz, R.-D., Kharchenko, N. V., Piskunov, A. E., Röser, S., \& Schilbach, E. 2015, A\&A, 581, A39

Skiff, B. A. 2014, VizieR Online Data Catalog, 1

Skrutskie, M. F., Cutri, R. M., Stiening, R., et al. 2006, AJ, 131, 1163

Taylor, M. B. 2005, in Astronomical Data Analysis Software and Systems XIV, eds. P. Shopbell, M. Britton, \& R. Ebert, ASP Conf. Ser., 347, 29

Tetzlaff, N., Neuhäuser, R., \& Hohle, M. M. 2011, MNRAS, 410, 190 van Leeuwen, F. 2007, A\&A, 474, 653

Zacharias, N., Finch, C. T., Girard, T. M., et al. 2013, AJ, 145, 44

Zacharias, N., Finch, C., Subasavage, J., et al. 2015, AJ, 150, 101 